\title{
ON THE MULLINS-SEKERKA MODEL FOR PHASE TRANSITIONS IN MIXTURES
}

\author{
$\mathrm{BY}$ \\ NATASA MILIC \\ Carnegie-Mellon University, Pittsburgh, Pennsylvania
}

\begin{abstract}
The Mullins-Sekerka model for dynamical phase transitions in twocomponent mixtures is considered. Global growth conditions for the phase regions and the interface are obtained from underlying conservation laws. A quasi-static model is formulated and the solutions are discussed for totally isolated mixtures.
\end{abstract}

Introduction. This paper presents a brief discussion of the mathematical model that was used by Mullins and Sekerka in a number of studies concerning phase transitions in multi-component materials. ${ }^{1}$ Recently it has been shown that this model arises as an approximation of a more general model which is appropriate to nonequilibrium phase transitions and totally compatible with thermodynamics. ${ }^{2}$ However, the approximate nature of the interface conditions proposed by Mullins and Sekerka affects the form of underlying conservation laws. It is our goal here to establish identities that can be viewed as conservation laws for the model and to investigate their implications on the growth of the interface and the phase regions. For specific conditions at the boundary of the mixture these identities lead to global growth conditions for the interfacial area and the phase volumes.

We are particularly interested in two types of boundary conditions:

(a) a completely isolated boundary on which there is no mass and no heat flux, and

(b) an isothermal boundary with no mass flux.

In both cases the conservation laws yield a Lyapunov function for the system that may be used to formulate appropriate equilibrium theories.

As an attempt to study phase transitions in which the interface moves slowly compared to the time scale of heat and mass diffusion in bulk we introduce a simplified, quasi-static model. Based on corresponding global growth conditions we were able

Received November 6, 1989.

${ }^{1}$ The Mullins-Sekerka model has served as a starting point for morphological stability analyses of interfaces using perturbation analysis (cf. Mullins and Sekerka [2] and Sekerka [3, 4, 7]).

${ }^{2}$ In $[9,10]$ Gurtin developed mathematical models for phase transitions governed exclusively by thermal diffusion. Following similar ideas Milic [11] has derived a model that accounts for effects of both transfer of heat and mass. For the sake of simplicity that model was restricted to mixtures with isotropic interfaces; a generalization to anisotropic interfaces can be patterned after Gurtin [10].

(C)1991 Brown University 
to characterize solutions for a mixture with a totally isolated boundary.

1. Basic equations. Conservation laws and growth conditions. For simplicity we assume that the material under consideration is composed of two constituents, referred to as the solute and the solvent. The composition of the mixture is then completely specified by $c$, the concentration of the solute. On the other hand, the melting temperature of the pure solvent may serve as a reference point for measuring $u$, the relative temperature of the mixture. ${ }^{3}$

We suppose that the material is contained in a fixed bounded region $\Omega$ in $\mathbb{R}^{n}$, $n=2,3$, with smooth boundary $\partial \Omega$. At each time $t, \Omega$ is divided by an interface $\mathrm{I}(t)$ into two regular subregions $\Omega_{i}(t), i=1,2$ :

$$
\begin{gathered}
\mathbf{I}(t)=\partial \Omega_{1}(t) \cap \partial \Omega_{2}(t), \\
\operatorname{int}\left(\Omega_{1}(t)\right) \cap \operatorname{int}\left(\Omega_{2}(t)\right)=\varnothing, \quad \Omega=\Omega_{1}(t) \cup \Omega_{2}(t),
\end{gathered}
$$

for all $t$. Regions $\Omega_{1}$ and $\Omega_{2}$ correspond to two distinct phases: phase 1, the solid phase, and phase 2 , the liquid phase.

Mullins and Sekerka suggested that the transport of heat and mass in the interior of the phase regions is governed by uncoupled diffusion equations. Comparing experimental diffusion rates in solids and liquids they proposed equal specific heats for the two phases and neglected the diffusion of the solute in the solid. Under these assumptions the bulk temperature and concentration satisfy the following equations:

$$
\begin{gathered}
a u^{\circ}=-\operatorname{div} \mathbf{q}, \quad c=c(\mathbf{x}) \quad \text { in } \Omega_{1}(t), \\
a u^{\circ}=-\operatorname{div} \mathbf{q}, \quad c^{\circ}=-\operatorname{div} \mathbf{h} \quad \text { in } \Omega_{2}(t),
\end{gathered}
$$

where

$$
\begin{array}{lc}
\mathbf{q}_{i}=-\mathbf{K}_{i} \operatorname{grad} u_{i}, & i=1,2, \\
\mathbf{h}_{2}=-\mathbf{H}_{2} \operatorname{grad} c_{2}, & \mathbf{h}_{1}=\mathbf{0},
\end{array}
$$

with $\mathbf{K}_{i}, i=1,2$, and $\mathbf{H}_{2}$ constant, symmetric, and positive definite matrices.

One expects that in a vicinity of the interface, the temperature and the concentration are affected by the motion of the interface. In the Mullins-Sekerka model this is reflected in the interface conditions: ${ }^{4}$

$$
\begin{gathered}
u_{1}=u_{2}, \\
u=-\alpha \gamma c_{2}+\beta \kappa, \quad c_{1} / c_{2}=\lambda, \\
{[\mathbf{q}] \cdot \mathbf{m}=L v, \quad \mathbf{h}_{2} \cdot \mathbf{m}=\gamma c_{2} v, \quad \text { on } \mathbf{I}(t),}
\end{gathered}
$$

\footnotetext{
${ }^{3}$ Here, the relative temperature $u=T-T_{m}$ is the difference between the absolute temperature $T$ and the melting temperature of the pure solvent $T_{m}$.

${ }^{4}$ It is tacitly assumed that the interface is in local equilibrium with the neighboring bulk material. Consequently, the temperature is continuous across the interface (cf. Gibbs [1]). On the other hand, at most jump discontinuities are allowed for the concentration $c$, the mass flux $\mathbf{h}$, and the heat flux $\mathbf{q}$ at the interface.
} 
with $\alpha, \beta, \lambda$, and $L$ strictly positive constants, $\lambda \neq 1$, and $\gamma=1-\lambda{ }^{5}$

Here we write $\mathbf{m}(\mathbf{x}, t)$ for the outer normal to $\partial \Omega_{1}(t) \cap \mathbf{I}(t), v(\mathbf{x}, t)$ for the component of the interfacial velocity in the direction of $\mathbf{m}(\mathbf{x}, t)$, and $\kappa(\mathbf{x}, t)$ for the sum of principal curvatures of the interface with $\kappa>0$ when the center of the curvature lies toward $\Omega_{1}(t)$.

The expression $[g]=g_{2}-g_{1}$ denotes the jump in quantity $g$ across the interface as the difference of limits $g_{i}, i=1,2$, of $g$ when the interface is approached from the interior of the phase regions. ${ }^{6}$

In addition to (1.6)-(1.8) we assume that the interface is either orthogonal to the boundary of $\Omega$ or the normal velocity $v$ vanishes, ${ }^{7}$

$$
v \mathbf{m} \cdot \mathbf{n}=0 \text { on } \partial \mathbf{I}(t),
$$

where the set $\partial \mathbf{I}$ corresponds to the intersection of the interface with the boundary of $\Omega$ and $\mathbf{n}(\mathbf{x}, t)$ denotes the outer normal to the boundary of $\Omega$.

Our derivation of the conservation laws and the global growth conditions is greatly simplified by introducing the function $c^{\wedge}$ :

$$
\widehat{c^{-}}(x, t):= \begin{cases}(1 / \lambda) c_{1}(\mathbf{x}, t) & \text { in } \Omega_{1}(t), \\ c_{2}(\mathbf{x}, t) & \text { in } \Omega_{2}(t),\end{cases}
$$

which is, in contrast to the concentration $c$, continuous across the interface (cf.


transforming $(1.2)_{2}$ into

$$
\widehat{c^{-}}(x)=c(\mathbf{x}) / \lambda \text { in } \Omega_{1} .
$$

From the bulk equations (1.2) and (1.3) and the jump conditions (1.8) we obtain the identities

$$
\begin{gathered}
\left\{L \operatorname{vol}\left(\Omega_{2}\right)+a \int_{\Omega} u\right\}^{\circ}=-\int_{\partial \Omega} \mathbf{q} \cdot \mathbf{n}, \\
\left(\int_{\Omega} c^{-}\right)^{\circ}-\int_{\mathbf{I}} \gamma c_{2} v=-\int_{\partial \Omega} \mathbf{h} \cdot \mathbf{n}
\end{gathered}
$$

that correspond to balance of energy and balance of mass, respectively. They are a simple consequence of the Divergence Theorem and the identities (A1) and (A2) stated in the Appendix.

In addition to (1.12) and (1.13) we find that

$$
\left\{\beta L \operatorname{area}(\mathbf{I})+(a / 2) \int_{\Omega} u^{2}+\alpha L \int_{\Omega} c^{-}\right\}^{\circ}=-|u|_{\mathbf{I}}-\int_{\partial \Omega} u \mathbf{q} \cdot \mathbf{n}-\alpha L \int_{\partial \Omega} \mathbf{h} \cdot \mathbf{n},
$$

\footnotetext{
${ }^{5}$ The parameter $\lambda=c_{1} / c_{2}$ is either strictly less or strictly greater than 1 , indicating that the solute either resists solidification and remains in higher concentration in the neighboring liquid $(\lambda<1)$ or is cooperative with solidification and assumes higher concentration in the solid $(\lambda>1)$. A strictly positive constant $L$ corresponds to the amount of heat released at the interface due to the phase transformation of the pure solvent (at $T=T_{m}$, i.e., $u=0$ and $c=0$ ). $L$ is usually referred to as the latent heat of fusion.

${ }^{6}$ Note, with no danger of confusion we use $g_{i}(x, t)$ to denote a/the value of the quantity $g$ at a point $x$ and time $t$ when $x$ is the interior of $\Omega_{i}(t)$, and $\mathrm{b} /$ the limit of $g$ as the point $x$ on the interface $\mathbf{I}(t)$ is approached from the interior of the phase region $\Omega_{i}(t)$.

${ }^{7}$ Condition (1.9), not originally present in the Mullins-Sekerka model, can be obtained from the thermodynamical treatment of two-phase materials (cf. Gurtin [9] and Milic [11]).
} 
where

$$
|u|_{\mathbf{I}}:=\int_{\Omega_{1}} \operatorname{grad} u \cdot \mathbf{K}_{1} \operatorname{grad} u+\int_{\Omega_{2}} \operatorname{grad} u \cdot \mathbf{K}_{2} \operatorname{grad} u
$$

and

$$
\begin{aligned}
& \left\{\int_{\Omega} \ln \left(\hat{\left.c^{-}+\varepsilon\right)}\right\}^{\circ}-\gamma \int_{\mathbf{I}} c_{2} v\left(c_{2}+\varepsilon\right)^{-1}\right. \\
& =-\int_{\partial \Omega}\left(c^{-}+\varepsilon\right)^{-1} \mathbf{h} \cdot \mathbf{n}+\int_{\Omega_{2}}(c+\varepsilon)^{-2} \operatorname{grad} c \cdot \mathbf{H}_{2} \operatorname{grad} c,
\end{aligned}
$$

for any $\varepsilon>0$.

Indeed, as $(1.7)_{1}$ is multiplied by $v$ and integrated over the interface it yields

$$
L \int_{\mathbf{I}} u v=-\alpha \gamma \int_{\mathbf{I}} c_{2} v+\beta \int_{\mathbf{I}} \kappa v .
$$

On the other hand, from $(1.8)_{1}$,

$$
L \int_{\mathbf{I}} u v=(a / 2)\left(\int_{\Omega} u^{2}\right)^{\circ}+|u|_{\mathbf{I}}+\int_{\partial \Omega} u \mathbf{q} \cdot \mathbf{n} .
$$

Thus, combining (1.18), (1.13), and (A3), relation (1.17) is transformed into (1.14).

To obtain (1.16) we first note that $c+\varepsilon$ is strictly positive for every $\varepsilon>0$. Condition $(1.8)_{2}$ is therefore equivalent to

$$
\gamma v\left(c_{2}+\varepsilon\right)^{-1} c_{2}=-\left(c_{2}+\varepsilon\right)^{-1} \mathbf{H}_{2} \operatorname{grad} c_{2} \cdot \mathbf{m} \text { on } \mathbf{I}(t) .
$$

From the bulk equations $(1.2)_{2}-(1.3)_{2}$ and (A6) we find that

$$
\begin{aligned}
-\int_{\mathbf{I}}\left(c_{2}+\varepsilon\right)^{-1} \mathbf{H}_{2} \operatorname{grad} c_{2} \cdot \mathbf{m}= & \int_{\partial \Omega}\left(\hat{c}^{-}+\varepsilon\right)^{-1} \mathbf{h} \cdot \mathbf{n}+\int_{\Omega_{2}}\left(\hat{c}^{-}+\varepsilon\right)^{-1}\left(\hat{c}^{-}\right)^{\circ} \\
& +\int_{\Omega_{2}}(c+\varepsilon)^{-2} \operatorname{grad} c \cdot \mathbf{h},
\end{aligned}
$$

where, because of the continuity of $c^{-}$across the interface, the second term on the right can be expressed as



The identity (1.16) thus follows directly from (1.19)-(1.21). In fact, by expanding $c_{2}=c_{2}+\varepsilon-\varepsilon$ in (1.16) and using the identity (A2) from the Appendix we arrive at an alternative form:

$$
\begin{aligned}
& \left\{\gamma \operatorname{vol}\left(\Omega_{2}\right)+\int_{\Omega} \ln \left(\hat{c}^{-}+\varepsilon\right)\right\}^{\circ}+\gamma \int_{\mathbf{I}} \varepsilon v\left(c_{2}+\varepsilon\right)^{-1} \\
& =-\int_{\partial \Omega}\left(c^{-}+\varepsilon\right)^{-1} \mathbf{h} \cdot \mathbf{n}+\int_{\Omega_{2}}(c+\varepsilon)^{-2} \operatorname{grad} c \cdot \mathbf{H}_{2} \operatorname{grad} c .
\end{aligned}
$$

When applied to specific boundary conditions relations (1.12)-(1.14) and (1.22) lead to the global growth conditions for the interfacial area and the phase volumes:

(I) Suppose that $\Omega$ has a totally isolated boundary:

$$
\mathbf{q} \cdot \mathbf{n}=0, \quad \mathbf{h} \cdot \mathbf{n}=0 \quad \text { on } \partial \Omega \text { for all } t .
$$


Then

$$
\begin{gathered}
\left\{L \operatorname{vol}\left(\Omega_{2}\right)+a \int_{\Omega} u\right\}^{\circ}=0, \\
\left\{\beta \operatorname{area}(\mathbf{I})+(a / 2) \int_{\Omega} u^{2}+\alpha L \int_{\Omega} c^{-}\right\}^{\circ}=-|u|_{\mathbf{I}} \leq 0, \\
\left\{\int_{\Omega} c\right\}^{\circ}=0,
\end{gathered}
$$

and, given $\varepsilon>0$,

$$
\begin{gathered}
\left\{\gamma \operatorname{vol}\left(\Omega_{2}\right)+\int_{\Omega} \ln \left(\widehat{c^{-}}+\varepsilon\right)\right\}^{\circ}+\gamma \int_{\mathbf{I}} \varepsilon v\left(c_{2}+\varepsilon\right)^{-1} \\
=\int_{\Omega_{2}}(c+\varepsilon)^{-2} \operatorname{grad} c \cdot \mathbf{H}_{2} \operatorname{grad} c
\end{gathered}
$$

(II) Suppose that $\Omega$ has an isothermal boundary across which there is no mass flux:

$$
u=u_{0}=\text { const }, \quad \mathbf{h} \cdot \mathbf{n}=0 \quad \text { on } \partial \Omega \text { for all } t .
$$

Then

$$
\begin{gathered}
\left\{-u_{0} \operatorname{vol}\left(\Omega_{2}\right)+\beta \operatorname{area}(\mathbf{I})+(a / 2 L) \int_{\Omega}\left(u-u_{0}\right)^{2}+\alpha \int_{\Omega} c^{-}\right\}^{\circ} \\
=-(1 / L)|u|_{\mathbf{I}} \leq 0, \\
\left\{\int_{\Omega} c\right\}^{\circ}=0,
\end{gathered}
$$

and (1.27) holds.

REMARK 1. We note that the quantities enclosed by \{\} in (1.25) and (1.29) do not increase with time. One may therefore regard stationary points of these quantities as equilibrium states for the system. For the derivation and discussion of corresponding variational problems we refer to Milic [11] which generalizes Gurtin's [9] results for the pure thermal case.

REMARK 2. The global growth conditions may further be used to make inferences concerning solutions of a simplified Mullins-Sekerka model that is appropriate to quasi-static phase transitions. In such situations the interface moves slowly compared to the time scale of heat and mass transfer in bulk and the Mullins-Sekerka model reduces to: ${ }^{8}$

$$
\begin{gathered}
\operatorname{div} \mathbf{q}=0, \quad c=c(\mathbf{x}) \quad \text { in } \Omega_{1}(t), \\
\operatorname{div} \mathbf{q}=0, \quad \operatorname{div} \mathbf{h}=0 \quad \text { in } \Omega_{2}(t) ; \\
{[\mathbf{q}] \cdot \mathbf{m}=L v, \quad \mathbf{h}_{2} \cdot \mathbf{m}=\gamma c_{2} v,} \\
u=-\alpha \gamma c_{2}+\beta \kappa, \quad c_{1} / c_{2}=\lambda \quad \text { on } \mathbf{I}(t), \\
\mathbf{q}_{i}=-\mathbf{K}_{i} \operatorname{grad} u_{i}, \quad i=1,2, \quad \mathbf{h}_{2}=-\mathbf{H}_{2} \operatorname{grad} c_{2} .
\end{gathered}
$$

\footnotetext{
${ }^{8}$ The quasi-static model (1.33)-(1.35) is obtained upon rescaling the quasi-linear model (1.2)-(1.8) (cf. Gurtin [9]). Because of the appearance of the interfacial velocity in the free-boundary conditions (1.33), the system (1.33)-(1.35) is rate dependent.
} 
The growth conditions corresponding to a totally isolated boundary (cf. (1.23)(1.26)) imply that, within the quasi-static theory, the phase volumes remain constant and the interfacial area does not increase:

$$
\begin{gathered}
\operatorname{vol}\left(\Omega_{2}\right)^{\circ}=-\operatorname{vol}\left(\Omega_{1}\right)^{\circ}=0, \\
\beta \operatorname{area}(\mathbf{I})^{\circ}=-|u|_{\mathbf{I}} \leq 0 .
\end{gathered}
$$

Also, given any $\varepsilon>0$,

$$
\gamma \int_{\mathbf{I}} \varepsilon v\left(c_{2}+\varepsilon\right)^{-1}=\int_{\Omega_{2}}(c+\varepsilon)^{-2} \operatorname{grad} c \cdot \mathbf{H}_{2} \operatorname{grad} c .
$$

If we regard the interface $\mathbf{I}(t)$ as a $C^{\infty}$-manifold that propagates smoothly so that the normal velocity $v(\mathbf{x}, t)$ is an infinitely differentiable $L^{1}$-function of $\mathbf{x}$ on the interface $\mathbf{I}(t)$,

$$
\int_{\mathbf{I}(t)}|v(\mathbf{x}, t)|<\infty \text { for each } t
$$

then we may further conclude that the concentration $c$ in the liquid phase of a totally isolated mixture must vanish identically

$$
c_{2} \equiv 0 \quad \text { in } \Omega_{2}(t),
$$

unless the interface is a sphere fixed in time. On the other hand, a spherical interface with radius $R$ does not move,

$$
v \equiv 0
$$

and the corresponding temperature and solute distributions are given by

$$
\begin{gathered}
u \equiv-\alpha c_{2} \pm \beta(1 / R), \\
c= \begin{cases}c_{1}(\mathbf{x}, t) & \text { in } \Omega_{1}(t), \\
c_{2}=C_{2}(t) & \text { in } \Omega_{2}(t) .\end{cases}
\end{gathered}
$$

This observation is a consequence of condition (1.38) and the Lemma stated in the Appendix.

Indeed, assume that $c_{2}$ is not identically zero in the liquid phase and, for the sake of concreteness, consider $\gamma>0$. Let

$$
f_{\varepsilon}:=\varepsilon v\left(c_{2}+\varepsilon\right)^{-1} \text { on } \mathbf{I}(t),
$$

for $\varepsilon>0$, and denote by $\mathbf{I}_{0}(t)$ a subset of $\mathbf{I}(t)$ on which $c_{2}(\cdot, t)$ vanishes. Since for each $\varepsilon>0$,

$$
\left|f_{\varepsilon}(\mathbf{x}, t)\right| \leq|v(\mathbf{x}, t)| \text { on } \mathbf{I}(t),
$$

and

$$
f_{\varepsilon}(\mathbf{x}, t) \rightarrow \begin{cases}0 & \text { on } \mathbf{I}(t) \backslash \mathbf{I}_{0}(t), \\ v(\mathbf{x}, t) & \text { on } \mathbf{I}_{0}(t),\end{cases}
$$

as $\varepsilon \rightarrow 0$, the Lebesgue Dominated Convergence Theorem implies that

$$
\lim _{\varepsilon \rightarrow 0} \int_{\mathbf{I}(t)} f_{\varepsilon}=\int_{\mathbf{I}_{0}(t)} v
$$


However, the Lemma applied to the system (1.31), (1.32), and (1.33) reveals that the set $\left\{\mathbf{x} \in \mathbf{I}_{0} \mid v(\mathbf{x}, t)>0\right\}$ cannot have a positive measure unless $c_{2} \equiv 0$. The integral over $\mathbf{I}_{0}$ in (1.47) is therefore nonpositive. This, in conjunction with (1.38) $(\gamma>0)$, shows that the concentration in liquid must be constant and the velocity must vanish for almost all points on $\mathbf{I}_{0}(t)$ :

$$
\begin{gathered}
v(\mathbf{x}, t)=0 \quad \text { a.e. on } \mathbf{I}_{0}(t), \\
c_{2}(\mathbf{x}, t)=C_{2}(t)>0 \text { in } \Omega_{2}(t) .
\end{gathered}
$$

In fact, from $(1.3)_{2}$,

$$
v(\mathbf{x}, t)=0 \quad \text { a.e. on } \mathbf{I}(t),
$$

and the interfacial area does not change (cf. (A5)),

$$
\operatorname{area}(\mathbf{I}(t))^{\circ}=0 \text {. }
$$

On the other hand, because of $(1.33)$ and the continuity of $u$ across the interface, the temperature is constant throughout the whole mixture,

$$
u=U(t) \text { in } \Omega \text {. }
$$

Consequently, the interface is a sphere fixed in time, with a radius $R$ determined by $(1.34)_{1}$ :

$$
\frac{1}{R}=\kappa(t)=\frac{1}{\beta} U(t)+\frac{\alpha}{\beta} \gamma C_{2}(t) .
$$

A completely analogous argument shows that the above statement is true when $\gamma<0$.

The preceding observation thus leads to the conclusion that, for a two-phase mixture with a completely isolated boundary and a nonspherical interface, the quasi-static theory reduces to the quasi-static theory for heat conduction in the absence of mass diffusion (cf. Gurtin [9]).

Appendix. Let $\Omega$ and $\Omega_{i}, i=1,2$, be regular regions in $\mathbb{R}^{n}$ described in Sec. 1 . For $g(\mathbf{x}, t)$, a vector- or a scalar-valued function on $\Omega \times \mathbb{R}^{+}$, with the jump across the interface $[g]=g_{2}-g_{1}$ we have the following standard identity:

$$
\left(\int_{\Omega} g\right)^{\circ}=\int_{\Omega_{1}(t)} g^{\circ}+\int_{\Omega_{2}(t)} g^{\circ}-\int_{\mathbf{I}(t)}[g] v
$$

for all time $t$.

A direct application of $(\mathrm{A} 1)$ to the function

yields

$$
g=\left\{\begin{array}{ll}
0 & \text { in } \Omega_{1}(t), \\
1 & \text { in } \Omega_{2}(t),
\end{array} \text { for all } t,\right.
$$

$$
\left(\operatorname{vol} \Omega_{2}\right)^{\circ}=-\int_{\mathbf{I}} v \text {. }
$$

On the other hand, a change in the interfacial area is determined as: ${ }^{9}$

$$
(\operatorname{area} \mathbf{I}(t))^{\circ}=-\int_{\mathbf{I}} \kappa v-\int_{\partial \mathbf{I}(t)} v \cot (\mathbf{m} \cdot \mathbf{n}) .
$$

\footnotetext{
${ }^{9}$ The identity (A3) is established by Gurtin, Struthers, and Williams [12].
} 
In our applications the last term in (A3) will vanish due to the condition

$$
v \mathbf{m} \cdot \mathbf{n}=0 \quad \text { on } \partial \mathbf{I} .
$$

Therefore,

$$
(\operatorname{area} \mathbf{I}(t))^{\circ}=-\int_{\mathbf{I}} \kappa v .
$$

Further, as an immediate consequence of the Divergence Theorem we have that

$$
\int_{\mathbf{I}}[\mathbf{g}] \cdot \mathbf{m}=\left(\int_{\partial \Omega}-\int_{\partial \Omega_{1}}-\int_{\partial \Omega_{2}}\right) \mathbf{g} \cdot \mathbf{n}
$$

for any smooth vector field $\mathbf{g}(\mathbf{x})$ defined on $\Omega$.

Here we state a simplified version of a result of Schmidt and Weck [6] concerning elliptic systems with mixed boundary conditions:

Lemma. ${ }^{10}$ Let $\Omega$ be a bounded domain in $\mathbb{R}^{n}$ and a function $c(\mathbf{x})$ be a nontrivial solution of the equation

$$
\operatorname{div}(A(\operatorname{grad} c))=0 \quad \text { in } \Omega,
$$

with $A$ a constant matrix, symmetric and positive definite. Suppose that for some open set $\mathscr{O} \subset \mathbb{R}^{n}$ :

(i) $\Gamma=\mathscr{O} \cap \partial \Omega$ is an $(n-1)$-dimensional $C^{\infty}$-manifold;

(ii) $A(\operatorname{grad} c) \cdot \mathbf{m}+b c=0$ on $\Gamma$,

where $b(\mathbf{x})$ is strictly positive and infinitely differentiable on $\Gamma$. Then $c$ vanishes on $\Gamma$ at most on a set of measure zero.

Acknowledgment. I express a deep gratitude to my advisor Morton E. Gurtin for his helpful guidance and close monitoring of this work. Special thanks to Carlos Kenig and Thomas Wolff for locating significant references. This work was supported by the Army Research Office and the National Science Foundation.

\section{REFERENCES}

[1] J. W. Gibbs, On the equilibrium of heterogeneous substances, Trans. Connecticut Acad. 3, 108-248 (1876), 343-524 (1878). Reprinted in The Scientific Papers of J. Willard Gibbs, Vol. 1, Dover, New York, 1961

[2] W. W. Mullins and R. F. Sekerka, Morphological stability of a particle growing by diffusion or heat flow, J. Appl. Phys. 34, 323-329 (1963)

[3] R. F. Sekerka, Morphological stability, J. Crystal Growth (3) 4, 71-81 (1968)

[4] R. F. Sekerka, Morphological stability, Crystal Growth: an Introduction, North-Holland, Amsterdam, 1973

[5] N. Weck, Uber das Prinzip der eindeutigen Fortsetzbarkeit in der Kontrolltheorie, Optimization and Optimal Control, Lecture Notes in Math., vol. 477, Springer-Verlag, Berlin, 1975, pp. 276-284

[6] E. J. P. Georg Schmidt and N. Weck, On the boundary behavior of solutions to elliptic and parabolic equations - with application to boundary control for parabolic equations, SIAM J. Control Optim. 16, 593-598 (1978)

[7] R. F. Sekerka, Morphological instabilities during phase transformations, Phase Transformations and Material Instabilities in Solids (M. E. Gurtin, ed.), Academic Press, New York, 1984

[8] M. E. Gurtin, On the theory of phase transitions with interfacial energy, Arch. Rational Mech. Anal. 87 187-212 (1985)

\footnotetext{
${ }^{10}$ Schmidt and Weck [6, Theorem 2.1]; see also Weck [5].
} 
[9] M. E. Gurtin, On the two-phase Stefan problem with interfacial energy and entropy, Arch. Rational Mech. Anal. 96, 199-241 (1986)

[10] M. E. Gurtin, Multiphase thermomechanics with interfacial structure: Heat conduction and capillary balance law (forthcoming)

[11] N. Milic, On the non-equilibrium phase-transitions in mixtures with interfacial structure, Ph.D. Thesis, Carnegie-Mellon University, Pittsburgh, PA, 1988

[12] M. E. Gurtin, A. Struthers, and W. O. Williams, A transport theorem for moving interfaces (forthcoming) 